\title{
Research progress on skin photoaging and oxidative stress
}

\author{
Xi Chen ${ }^{1}$, Chunsheng Yang ${ }^{2}$, Guan Jiang ${ }^{1}$
}

${ }^{1}$ Department of Dermatology, Affiliated Hospital of Xuzhou Medical University, Xuzhou, China

2Department of Dermatology, the Affiliated Huai'an Hospital of Xuzhou Medical University, the Second People's Hospital of Huai'an, Huai'an, China

Adv Dermatol Allergol 2021; XXXVIII (6): 931-936

DOI: https://doi.org/10.5114/ada.2021.112275

\begin{abstract}
Skin photoaging, which is a kind of exogenous aging, refers to skin elasticity degradation, skin roughening, and wrinkle formation processes because of cascading reactions of a series of kinases after growth factor receptors and cytokine receptors are activated on a cell surface under the UV effect. An extensively recognized skin photoaging mechanism is free radical-oxidative stress concept, proposed by Sohal who represents the authority of the US aging studies. Over the past decade, many new developments in the oxidative stress mechanism have been achieved in terms of the occurrence, development, prevention, and treatment of photodamage.
\end{abstract}

Key words: UV, photoaging, oxidative stress, photodamage.

\section{Introduction}

Skin aging, a complicated process and the most intuitive external presentation of the aging of the organism, is generally divided into endogenous aging and exogenous aging [1]. The former belongs to natural aging, which refers to procedural aging caused by irresistible factors in the body, and the degree of aging is slow. Conversely, the latter is mostly caused by environmental factors, such as solar radiation, cigarette smoke or other environmental pollution factors [2]. These factors increase endogenous aging. The major factor of exogenous skin aging, also known as photoaging, is exposure to UV radiation [3-5]. Its main clinical manifestations are dry and rough skin on the exposed part, with deepened wrinkles, cutis laxa, angiotelectasis, and hyperpigmentation [6]. Epidemiological surveys and studies have verified that the genesis of many skin diseases and skin tumours is related to skin photoaging. Skin photoaging not only affects people's pursuit of beauty but also endangers health, which has gradually drawn concerns from people in recent years.

\section{UV and photoaging}

UV from sunlight is divided into short-wave UV (UVC, 100-290 nm), medium-wave UV (UVB, 290-320 nm), and long-wave UV (UVA, 320-400 nm) (Figure 1). With the ozone layer in the atmosphere, UVC is nearly completely absorbed, and UV reaching the ground is mainly UVA and
UVB [7]. A high amount of UVB radiation reaches our skin direct sunshine, just at noon, when the sun is seemingly positioned at the top of the beting down. However, UVA radiation is almost never absorbed when it passes through the Earth's atmosphere, so it is abundantly present in solar radiation all day long. Therefore, the degree of UVA exposure of the skin is much larger than that of UVB exposure under normal conditions [5]. Even though 95\% and $<5 \%$ of UV in sunlight radiation are UVA and UVB, respectively, the bioactivity of UVA is much weaker than that of UVB, that is $1 / 1,000$ of UVB. Therefore, UV, which causes skin erythema, DNA damage, and skin cancer, is mainly UVB, whereas UVA almost does not harm the human body. Similar to UVB, intense UVA irradiation can generate erythema and vascular damage, and it even induces more serious changes than UVB does. The amount of UVA needed to generate this effect is 1,000 times greater than that of UVB. The skin penetrating power of UVA is higher than that of UVB, so UVA1 (340-400 nm) can penetrate the corium layer more deeply, thus causing damage to collagens and elastic fibres in dermal tissues. Therefore, the effect of UVA on the pathogenesis of skin photoaging has been intensively investigated [8-10].

\section{Oxidative stress and photoaging}

In daily life, humans are constantly exposed to oxidants either from endogenous metabolic processes or

Address for correspondence: Guan Jiang PhD, Department of Dermatology, Affiliated Hospital of Xuzhou Medical University, Xuzhou 221002, China, e-mail: dr.guanjiang@xzhmu.edu.cn Received: 5.10.2019, accepted: 19.12.2019. 


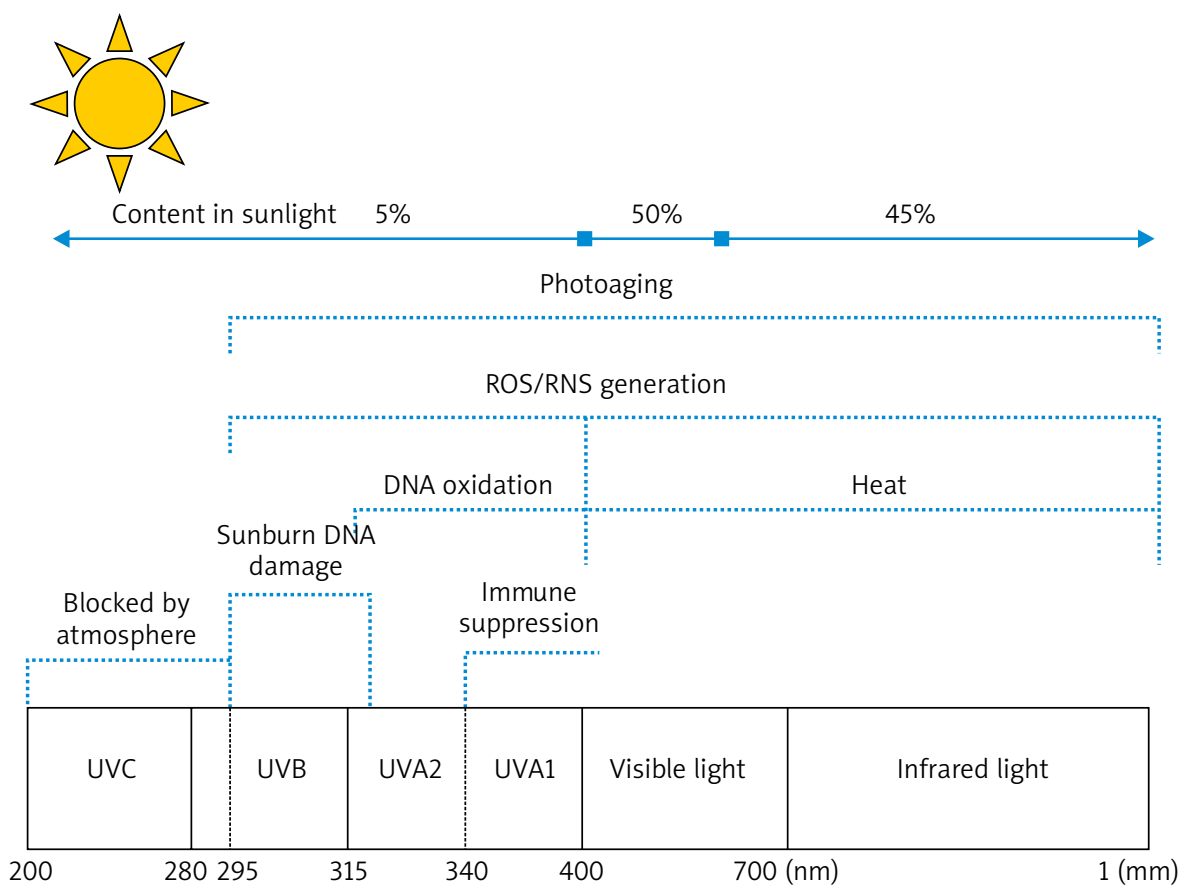

Figure 1. Mechanism of skin photoaging caused by UVA and UVB. UVA (315-400 nm): UVA penetrates the corium layer more deeply, with the strongest ability of generating reactive oxygen species (ROS) and lipid peroxidases, thereby causing damage to collagens and elastic fibres in dermal tissues. UVB (280-315 nm): UVB acts on the epidermis and causes skin erythema, skin tumours, and immunosuppression. UVC (100-280 nm): UVC is nearly completely absorbed by the ozone layer in the atmosphere

exogenous environmental pollutants. A strong antioxidant system has gradually formed in the human body to maintain redox homeostasis. Oxidative stress occurs when the oxidant/antioxidant balance is destroyed $[11,12]$. It is generally believed that oxidative stress has a significant effect on extrinsic skin aging, and studies point out that reactive oxygen species (ROS) is one of the major contributors (Figure 2) [1]. In the mitochondria, ROS is continuously produced as a side product in the electron transport chain of aerobic metabolism, and regarded as the main factor of endogenous aging. Keratinocytes and fibroblasts are the main cells that produce ROS in the skin. A ROS is predominantly formed by adding an electron to each oxygen molecule $\left(\mathrm{O}_{2}\right)$ in the mitochondria, from a ground state oxygen to the reactive superoxide anion radical $\left(\mathrm{O}_{2}\right)$. Excessive solar radiation on the skin induces the production of large amounts of ROS and reduces the production of peroxidase and glutathione reductase. Many studies have shown that ROS produced by keratinocytes and fibroblasts exhibit a dose-dependent effect on the amount of UV radiation [13-18]. Excessive UV radiation can cause direct skin tissue damage by attacking proteins, lipids, and DNA; it can also oxidize macromolecular substances, especially lipids and DNA, and influence a series of signal pathways to cause indirect skin damage through ROS-mediated oxidative stress reactions $[12,13,15,19]$.

\section{Protein oxidation}

ROS-induced oxidative damage to elastin and collagen in the dermis can lead to changes in protein conformation, thereby affecting the mechanical properties of the skin. In particular, histidine and lysine residues are the main targets of oxidative modification and can be converted to 2-oxo histidine and aminoadipate semialdehyde, respectively. Oxidative modifications of the relevant side chains of proteins or enzymes can result in a change in their properties and functions. Correspondingly, the metabolic function of the skin is affected, and photoaging is promoted $[5,20]$.

\section{Lipid oxidation}

An acyl double-bond reaction between ROS and unsaturated fatty acids can generate lipid peroxidases [14, 15, 21, 22]. Marionnet et al. [15] detected 8-IsoPs, a lipid peroxidase marker, in a skin model culture medium rebuilt in vitro after UVA1 irradiation and found that dosage dependence is significantly enhanced. This process has been verified through studies on the photosensitive reaction of lipids through large monolayer vesicles. Lipid peroxidase can form a hydrophilic pore canal on biological membranes to cause damage. The permeability of the damaged cell membrane or mitochondrial membrane increases, and the damage of oxidative stress to cells or 
adjacent cells aggravates. At the same time, inflammatory reactions also exacerbate, resulting in angiotelectasis [23]. The inflammatory response of the skin manifests as erythema. Water in blood vessels diffuses between tissues to cause swelling or accumulates in the skin to form vesicles. All these phenomena provide conditions for the genesis of photodamage-related diseases, such as sunburn, photoaging, and polymorphic light eruption $[14,21]$.

\section{DNA damage}

UV can directly damage DNA and generate a pyrimidine dimer and a 6-4 photoproduct [13, 24, 25]. A large amount of UV radiation-generated ROS can cause oxidative damage to nuclear DNA and produce a large amount of thymine glycol and 8-hydroxyl guanine through single base or purine modification, inter-chain crosslinking, DNA-protein crosslinking, and depurination or pyrimidine site formation $[13,24,26]$. ROS is mainly generated through aerobic metabolism or enzymatic reaction in mitochondria. Mitochondrial DNA (mtDNA), an extranuclear genetic material that is close to the ROS-producing part, lacks protective histones, and has poor reparability. Consequently, mtDNA can experience mutation more easily than nuclear DNA. This damage in mtDNA leads to defects in the electron transport chain and oxidative phosphorylation, ultimately resulting in reduced ATP production. Impaired oxidative phosphorylation also increases singlet oxygen generation, which in turn leads to more mtDNA mutations [27]. Mutated mtDNA replication is obviously enhanced in the photodamaged skin entering an oxidative stress state [28], indicating that mtDNA can also be damaged by ROS generated via UV irradiation. Under normal circumstances, the cancer suppressor gene p53 arrests the cell cycle in the G1 phase and repairs the mutated gene by DNA repairase in time before replication. If the gene damage is so severe that it cannot be adequately repaired, p53 can mediate the apoptosis of damaged cells by regulating the transcription of the apoptotic genes Bax and $\mathrm{Bcl}-2$. This process is important in DNA damage repair. However, long-term UV exposure can cause p53 gene mutations in epidermal keratinocytes, cells exhibit tolerance to apoptosis, the mitochondria experience dysfunction, and DNA carrying mutant genes enter the cell division cycle under an unrepaired circumstance, thereby causing malignant cell proliferation and metabolic disorders and promoting the genesis of photo-related skin tumours, such as squamous cell carcinoma (SCC), basal cell carcinoma (BCC), and melanoma [24, 28, 29]. Langerhans cells are important antigen-presenting cells in the epidermis. UV can reduce the number and antigen-presenting function of Langerhans cells, which induce immunosuppression and cause skin tumours to escape the immune surveillance of the body [30].

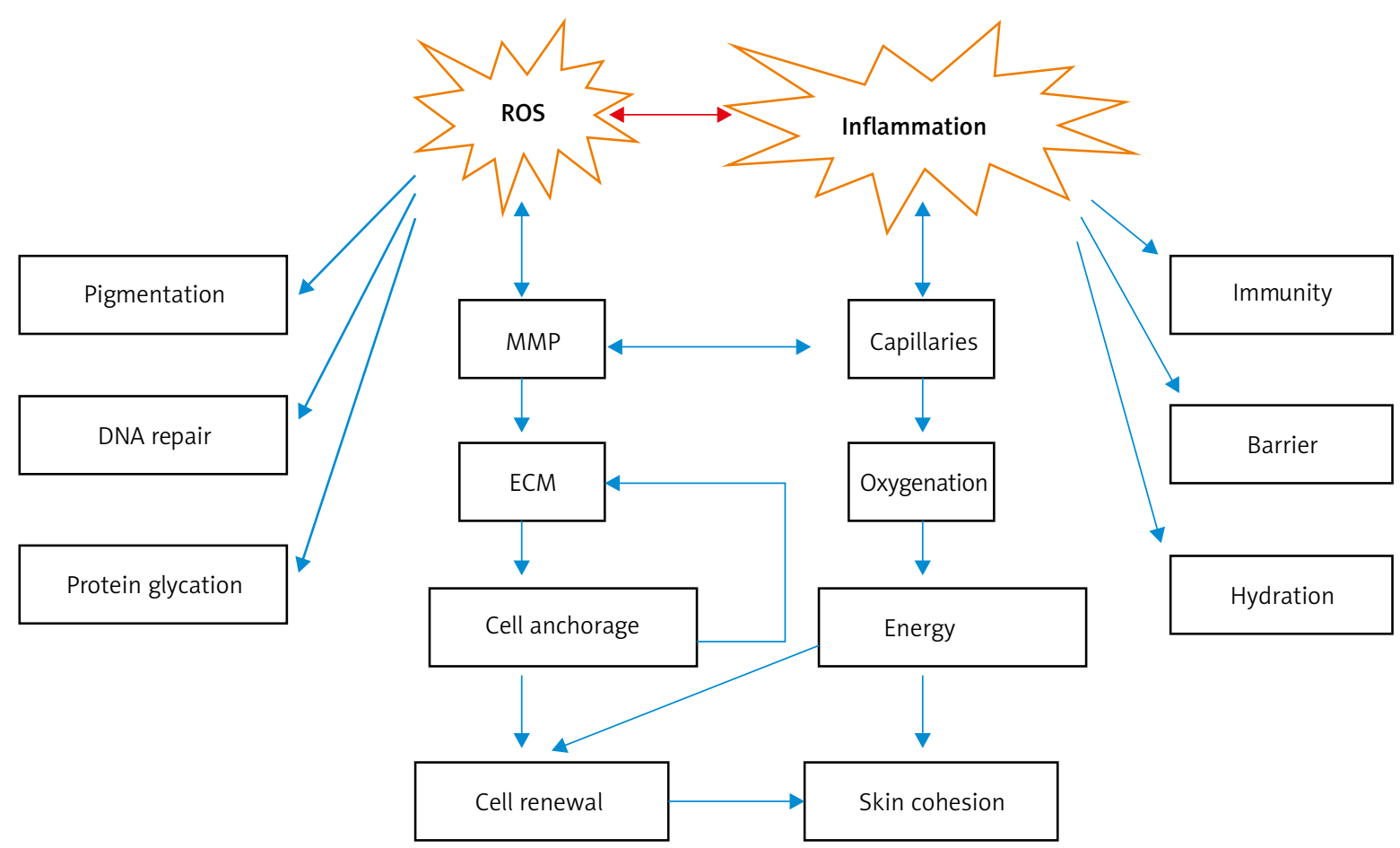

Figure 2. Series of changes caused by ROS and inflammation. ROS and inflammation can cause pigmentation and changes in matrix metalloproteinase activity, extracellular matrix components, skin immunity, and barrier function 


\section{Influences of skin photodamage and oxidative stress on signal pathways}

In oxidative stress, as a second messenger, ROS can activate relevant signal transduction pathways participating in inflammatory damage and tumorigenesis to further regulate the expression of downstream genes and stimulate various processes, such as skin damage, aging, and photo-induced cancer. Photodamage is mainly related to the following signal pathways.

\section{Activation of the signal pathways of mitogen- activated protein kinase}

UV-induced ROS can promote the phosphorylation of an epidermal growth factor receptor to trigger the cascading reaction of mitogen-activated protein kinases (MAPKs). MAPKs include three main protein phosphorylation cascades, namely, extracellular signalregulated kinase (ERK), C-Jun N-terminal kinase (JNKS), and p38MAPK. A large amount of ROS can be generated after the skin is exposed to UV irradiation, thereby activating JNK and p38MAPK pathways and transferring ERK1/2, JNK and p38MAPK to the nucleus. It can trigger protein-1 (AP-1) activation and upregulate cyclooxygenase-2 (COX-2) gene expression [31, 32]. As a result, the secretion of cytokines, such as interleukin(IL)-10, IL-8, vascular endothelial factor, and prostaglandin G2, increases to further promote processes, such as immunosuppression, inflammatory reaction, proliferation, differentiation and angiogenesis [23, 33, 34]. This process not only accelerates photoaging progression but also facilitates tumour cell infiltration and results in the invasion and metastasis of skin tumours, such as SCC [17, 35, 36]. ROS generated by UV are also indirectly responsible for the release of ceramides and arachidonic acid because of an increasing cell membranes permeability. An increase in the ceramide pool in the epidermis can trigger further AP-1 activation and is directly responsible for the initiation of keratinocyte apoptosis. In turn, arachidonic acid is converted by cyclooxygenase into prostaglandins, which are responsible for attracting lymphocytes to the area of damaged membranes. AP-1 induces matrix metalloproteinase (MMP) and cathepsin K via transcriptional upregulation, thereby increasing the degradation of dermal collagens, elastin, and matrix and aggravating photoaging $[17,18,21,24-26,37,38]$. As a result, the skin grows deep wrinkles, loses its elasticity, becomes dry, sag, and rough [39]. Furthermore, increased ROS levels can promote the upregulation of the expression of the apoptins Bax and p53 and facilitate the phosphorylation of p38MAPK. Conversely, the expression of the anti-apoptotic protein Blc-2 is downregulated [28]. A pro-apoptotic protein is transferred from the cytoplasm to the mitochondria so that cytochrome $\mathrm{C}$ is released to activate the downstream effector cysteine aspartic protease and induce cell apoptosis. What is more, UV-induced ROS can directly release cytochrome C from the mitochondria to the cytoplasm in the early stage of apoptosis. In addition, the inflammatory reaction is enhanced, and the skin exhibits acute photodamage manifestations, such as erythema, vesicle formation, and swelling, within a short period. Continuous exposure to UV contributes to the genesis of chronic actinic dermatitis, actinic keratosis, and SCC [24-26].

\section{Activation of the pathways of nuclear transcription factors}

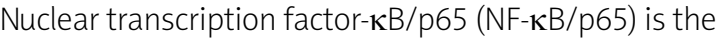
major transcription factor that plays a regulatory role in immune responses and inflammatory processes [40]. In normal cells, NF- $\kappa B$ combines with inhibitive $\kappa B(\mid \kappa B)$ to form a compound restricted in the cytoplasm. After UV irradiation, a large amount of ROS is produced in cells, thereby activating $l_{\kappa} B$ kinase to phosphorylate and degrade $I_{\kappa} B$ protein. NF- $\kappa B$ is then activated and transferred to the nucleus and combines encoding genes related to inflammation, proliferation, and tumorigenesis so that the expression of pro-inflammatory factors such as IL-8 and COX-2 and the transcription of cyclin D1 and MMP are upregulated. Cell proliferation and angiogenesis subsequently increase [11, 41]. MMPs degrade collagen fibres and elastic fibres, causing sagging and wrinkles in the skin. MMPs can also degrade the extracellular matrix (ECM) to cause cell migration, which is related to tumour metasta-

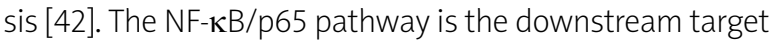
location of the MAPK signal transduction pathway. The NF$\kappa \mathrm{B} / \mathrm{p} 65$ pathway and the MAPK pathway present reciprocal chiasma and collaboration and jointly participate in photoaging and photo-induced cancer [17, 24, 28, 29].

\section{Inhibition of nuclear transcriptional \\ factor E2-related factor 2 signal pathway}

Nuclear transcriptional factor E2-related factor 2 (Nrf2), which is an important intracellular transcriptional factor regulating antioxidant stress, can protect the skin from oxidative stress damage caused by UV. Under normal physiological conditions, Nrf2 combines its profiling Drosophila actin binding protein (Keap) 1 to form a compound in the cytoplasm. Keap1 interacts with other proteins and plays a key role in regulating Nrf2 localization and degradation. Keap1 acts as an adaptor of the ubiquitin ligase E3 complex, resulting in the proteasomal degradation of $\mathrm{Nrf2}$. In the cysteine-rich intervening region, some cysteine residues, especially Cys 151, Cys 273 and Cys288, are extremely sensitive to oxidation, they are also required for Nrf2 binding [43]. When low-dosage UV induces ROS to generate and incur oxidative stress, these cysteine residues are modified, and Keap1 loses its ability to bind to Nrf2, thereby allowing Nrf2 to escape degradation. After being dissociated from Keap1, Nrf2 transfers to the nucleus from the cytoplasm. Then, Nrf2 identifies and combines with an antioxidant response element (ARE) 
in the II-phase genetic promoter region to activate the Nrf2/ARE signal pathway, promote the expression of intracellular antioxidant protective genes and upregulate the transcriptional levels of II-phase detoxifying enzymes and antioxidase $[44,45]$. Cellular protection-related antioxidant enzymes induced by Nrf2, mainly include glutathione S-transferase (GST), nicotinamide quinone oxidoreductase (NQ01), UDP-glucuronosyltransferases (UGT), $\gamma$-glutamyl glutamate cysteine ligase $(\mathrm{GCL})$, heme oxygenase-1 (HO-1), glutathione reductase (GR), catalase (CAT) and superoxide dismutase (SOD) [46, 47]. However, after high-dosage UV irradiation, ROS increases abnormally. Consequently, Nrf2 becomes inactivated and cannot transfer to the nucleus; the Nrf2-Keapl-ARE signal pathway is inhibited; the Nrf2 expression level is reduced; antioxidase or antioxidant levels decline; more ROS further accumulate; and signal pathways, such as MAKPs and NF- $\mathrm{B}$, are activated, resulting in dermal matrix degradation, cancer suppressor gene activation, and cell apoptosis. Acute photodamage, chronic photoaging, SCC, and BCC finally occur $[22,48,49]$.

\section{Conclusions}

Skin aging is a part of the body's aging and the most easily identifiable aging type. Single endogenous skin aging is absent and usually reinforced by exogenous factors. Skin aging is related to not only the appearance of the human body, but also health problems. A thorough understanding of the general aging process is critical to preventing and managing skin aging. ROS is likely the main cause of exogenous aging, can induce damage to biological macromolecules (DNA/proteins/lipids) and can regulate the transcription of several signalling pathways. MMP is another important molecule related to ROS in skin aging, causing damage to ECM homeostasis, which promotes wrinkle formation. Our summary of the molecular mechanisms involved in photoaging and oxidative stress is still limited, and further studies are needed to provide new ideas for preventing exogenous skin aging, especially photoaging.

\section{Acknowledgments}

This research was supported by the National Natural Science Foundation of China (Nos. 81572976, 81872493, 81803151), the China Postdoctoral Science Foundation (Nos. 2016M590505, 2017T100407), the Jiangsu Provincial Medical Talent Foundation, the 'Six Talent Peaks' Project of Jiangsu Province (Nos. WSW-074, WSN-254), and the Science and Technology Project of Huai'an city (No. HAB201812).

\section{Conflict of interest}

The authors declare no conflict of interest.

\section{References}

1. Poljšak B, Dahmane RG, Godić A. Intrinsic skin aging: the role of oxidative stress. Acta Dermatovenerol Alp Pannonica Adriat 2012; 21: 33-6.

2. Krutmann J, Schikowski T, Hüls A, et al. Environmentally induced (extrinsic) skin aging. Hautarzt 2016; 67: 99-102.

3. Han A, Chien AL, Kang S. Photoaging. Dermatol Clin 2014; 32: 291-9.

4. Bosch R, Philips N, Suárez-Pérez JA, et al. Mechanisms of photoaging and cutaneous photocarcinogenesis, and photoprotective strategies with phytochemicals. Antioxidants 2015; 4: 248-68.

5. Kammeyer A, Luiten RM. Oxidation events and skin aging. Ageing Res Rev 2015; 21: 16-29.

6. Zouboulis CC, Hoenig LJ. Skin aging revisited. Clin Dermatol 2019; 37: 293-5.

7. Kim BH, Choi MS, Lee HG, et al. Photoprotective potential of Penta-O-Galloyl- -DGlucose by targeting NF-B and MAPK signaling in UVB radiation-induced human dermal fibroblasts and mouse skin. Mol Cells 2015; 38: 982-90.

8. Bäumler W, Regensburger J, Knak A, et al. UVA and endogenous photosensitizers - the detection of singlet oxygen by its luminescence. Photochem Photobiol Sci 2012; 11: 107-17.

9. Lan CE, Hung YT, Fang AH, et al. Effects of irradiance on UVAinduced skin aging. J Dermatol Sci 2019; 94: 220-8.

10. Wenk J, Btenneisen P, Meewes C, et al. UV-induced oxidative stress and photoaging. Curr Probl Dermatol 2001; 29: 83-94.

11. Afnan Q, Kaiser PJ, Rafiq, et al. Glycyrrhizic acid prevents ultraviolet-b induced photodamage: a role for mitogenactivated protein kinases, nuclear factor kappa B and mitochondrial apoptotic pathway. Exp Dermatol 2016; 25: 440-6.

12. Wölfle U, Seelinger G, Bauer G, et al. Reactive molecule species and antioxidative mechanisms in normal skin and skin aging. Skin Pharmacol Physiol 2014; 27: 316-32.

13. Romanhole RC, Ataide JA, Moriel P, et al. Update on ultraviolet $A$ and $B$ radiation generated by the sun and artificial lamps and their effects on skin. Int J Cosmet Sci 2015; 37: 366-70.

14. Polefka TG, Meyer TA, Agin PP, et al. Cutaneous oxidative stress. J Cosmet Dermatol 2012; 11: 55-64.

15. Marionnet C, Pierrard C, Golebiewski C, et al. Diversity of biological effects induced by longwave UVA rays (UVA1) in reconstructed skin. PLoS One 2014; 9: e105263.

16. Zhong J, Li L. Skin-derived precursors against UVB-induced apoptosis via Bcl-2 and Nrf2 up-regulation. Biomed Res Int 2016; 2016: 6894743.

17. Liu Y, Chan F, Sun H, et al. Resveratrol protects human keratinocytes $\mathrm{HaCaT}$ cells from UVA-induced oxidative stress damage by downregulating Keap1 expression. J Photochem Photobiol B 2012; 109: 34-41.

18. Terra VA, Souza-Neto FP, Pereira RC, et al. Time-dependent reactive species formation and oxidative stress damage in the skin after UVB irradiation. J Photochem Photobiol B 2012; 109: 34-41.

19. Nys K, Van Laethem A, Michiels C, et al. A p38 (MAPK)/HIF-1 pathway initiated by UVB irradiation is required to induce Noxa and apoptosis of human keratinocytes. J Invest Dermatol 2010; 130: 2269-76.

20. Reeg S, Grune T. Protein oxidation in aging: does it play a role in aging progression? Antioxid Redox Signal 2015; 23: 239-55.

21. Itri R, Junqueira HC, Mertins O, et al. Membrane changes under oxidative stress: the impact of oxidized lipids. Biophys rev 2014; 6: 47-61. 
22. Yoo OK, Choi BY, Park JO, et al. Ethanol extract of cirsium japonicum var. ussuriense kitamura exhibits the activation of nuclear factor erythroid 2-related factor 2-dependent antioxidant response element and protects human keratinocyte HaCaT cells against oxidative DNA damage. J Cancer Prev 2016; 21: 66-72

23. Chung JH, Eun HC. Angiogenesis in skin aging and photoaging. J Dermatol 2007; 34: 593-600.

24. Beani JC. Ultraviolet A-induced DNA damage: role in skin cancer. Bull Acad Natl Med 2014; 198: 273-95.

25. Tewari A, Sarkany RP, Young AR. UVA1 induces cyclobutane pyrimidine dimers but not 6-4 photoproducts in human skin in vivo. J Invest Dermatol 2012; 132: 394-400.

26. Cadet J, Wagner R. DNA base damage by reactive oxygen species, oxidizing agents, and UV radiation. Cold Spring Harb Perspect Biol 2013; 5: pii: a012559.

27. Wang CH, Wu SB, Wu YT, Wei YH. Oxidative stress response elicited by mitochondrial dysfunction: implication in the pathophysiology of aging. Exp Biol Med 2013; 238: 450-60.

28. Birch-Machin MA, Swalwell H. How mitochondria record the effects of UV exposure and oxidative stress using human skin as a model tissue. Mutagenesis 2010; 25: 101-7.

29. Rebel HG, Bodmann CA, van de Glind GC, et al. UV-induced ablation of the epidermal basal layer including p53-mutant clones resets UV carcinogenesis showing squamous cell carcinomas to originate from interfollicular epidermis. Carcinogenesis 2012; 33: 714-20.

30. Damian DL, Matthews YJ, Phan TA, et al. An action spectrum for ultraviolet radiation-induced immunosuppression in humans. Br J Dermatol 2011; 164: 657-9.

31. Korbecki J, Baranowska-Bosiacka I, Gutowska I, Chlubek D. The effect of reactive oxygen species on the synthesis of prostanoids from arachidonic acid. J Physiol Pharmacol 2013; 64: 409-21.

32. Xu Q, Hou W, Zheng Y, et al. Ultraviolet A-induced cathepsin expression is mediated via MAPK/AP-1 pathway in human dermal fibroblasts. PLoS One 2014; 9: e102732.

33. Mutou $Y$, Tsukimoto $M$, Homma T, et al. Immune response pathways in human keratinocyte ( $\mathrm{HaCaT}$ ) cells are induced by ultraviolet B via p38 mitogen-activated protein kinase activation. J Health Sci 2010; 56: 675-83.

34. Wölfle U, Seelinger G, Bauer G, et al. Reactive molecule species and antioxidative mechanisms in normal skin and skin aging. Skin Pharmacol Physiol 2014; 27: 316-32.

35. Afnan Q, Kaiser PJ, Rafiq, et al. Glycyrrhizic acid prevents Ultraviolet-B induced photodamage: a role for mitogenactivated protein kinases, nuclear factor kappa B and mitochondrial apoptotic pathway. Exp Dermatol 2016; 25: 440-6.

36. Halliday GM, Byrne SN, Damian DL. Ultraviolet A radiation: its role in immunosuppression and carcinogenesis. Semin Cutan Med Surg 2011; 30: 214-21.

37. Hsu LY, Nien CY, Huang WM, et al. Synthesis and protective effects of bis\{4-[N,N-di-(carboxymethyl)amino]phenoxy\}alkane derivatives on UVA- induced production of MMP-1 in human skin fibroblasts. Chem Pharm Bull 2014; 62: 867-74.

38. Freitas-Rodríguez S, Folgueras AR, López-Otín C. The role of matrix metalloproteinases in aging: tissue remodeling and beyond. Biochim Biophys Acta Mol Cell Res 2017; 1864 2015-25.

39. Amano S. Characterization and mechanisms of photoageing-related changes in skin. Damages of basement membrane and dermal structures. Exp Dermatol 2016; 3: 14-9.

40. Morgan MJ, Liu ZG. Crosstalk of reactive oxygen species and NF- $\mathrm{B}$ s signaling. Cell Res 2011; 21: 103-15.
41. Buelna-Chontal M, Zazueta C. Redox activation of Nrf2 \& NF-кB: a double end sword? Cell Signal 2013; 25: 2548-57.

42. Nys K, Van Laethem A, Michiels C, et al. A p38 (MAPK)/HIF-1 pathway initiated by UVB irradiation is required to induce Noxa and apoptosis of human keratinocytes. J Invest Dermatol 2010; 130: 2269-76.

43. Sajadimajd S, Khazaei M. Oxidative stress and cancer: the role of Nrf2. Curr Cancer Drug Targets 2018; 18: 538-57.

44. Krajka-Kuźniak V, Paluszczak J, Baer-Dubowska W. The Nrf2ARE signalling pathway: an update on its regulation and possible role in cancer prevention and treatment. Pharmacol Rep 2017; 69: 393-402.

45. Kovac S, Angelova PR, Holmström KM, et al. Nrf2 regulates ROS production by mitochondria and NADPH oxidase. Biochim Biophys Acta 2015; 1850: 794-801.

46. Zhu H, Jia Z, Zhang L, et al. Thioredoxin reductase in human hepatoma cells is transcriptionally regulated by sulforaphane and other electrophiles via an antioxidant response element. Exp Biol Med 2008; 233: 463-74.

47. Reisman SA, Lee CY, Meyer CJ, et al. Topical application of the synthetic triterpenoid RTA 408 activates Nrf2 and induces cytoprotective genes in rat skin. Arch Dermatol Res 2014; 306: 447-54.

48. Gruber F, Mayer H, Lengauer B, et al. NF-E2-related factor 2 regulates the stress response to UVA-1-oxidized phospholipids in skin cells. FASEB J 2010; 24: 39-48.

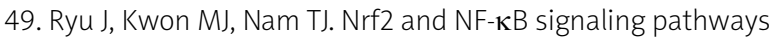
contribute to porphyra-334-mediated inhibition of UVAinduced inflammation in skin fibroblasts. Mar Drugs 2015; 13: 4721-32. 